\title{
Mechanisms for the induction of autoimmunity by infectious agents
}

\author{
Kai W. Wucherpfennig \\ Department of Cancer Immunology and AIDS, Dana-Farber Cancer Institute and \\ Department of Neurology, Harvard Medical School, Boston, Massachusetts, USA \\ Address correspondence to: Kai W. Wucherpfennig, Department of Cancer Immunology and AIDS, \\ Dana-Farber Cancer Institute, Room D1410, 44 Binney Street, Boston, Massachusetts 02115, USA. \\ Phone: (617) 632-3086; Fax: (617) 632-2662; E-mail: Kai_Wucherpfennig@dfci.harvard.edu.
}

J. Clin. Invest. 108:1097-1104 (2001). DOI:10.1172/JCI200114235.

\begin{abstract}
Activation and clonal expansion of autoreactive lymphocytes is a critical step in the pathogenesis of autoimmune diseases. In experimental models of autoimmunity, disease can be transferred by activated, but not resting, autoreactive $T$ cells (1), indicating that activation of autoreactive $T$ cells is required for the development of autoimmune diseases. Infectious agents have long been considered as possible culprits in the activation of autoreactive T cells. Mechanisms by which an infection can lead to an autoimmune process have been examined in experimental animal models, and these concepts as well as their relevance to human diseases will be discussed here.
\end{abstract}

\section{Basic mechanisms for the induction of autoimmunity by pathogens}

In general terms, mechanisms based on microbial products - such as peptides or superantigens - need to be distinguished from mechanisms based on the inflammatory setting that results from an infection. Infection can also result in lymphocyte activation when intracellular signaling pathways are manipulated by lymphotropic viruses (Table 1). Peptides from microbial proteins that have sufficient structural similarity with self-peptides can activate autoreactive $T$ cells, a mechanism that is referred to as molecular mimicry (2-12). Microbial superantigens activate large numbers of $\mathrm{T}$ cells that express particular $V \beta$ gene segments, and a subpopulation of these activated cells can be specific for a self-antigen (13-17). The inflammatory setting that results from a viral or bacterial infection leads to local activation of antigen-presenting cells and can result in enhanced processing and presentation of self-antigens present at that site. In chronic autoimmune diseases a similar process can result in the activation and expansion of $T$ cells with additional specificities, a process referred to as epitope spreading $(18,19)$. The inflammatory setting may also promote the expansion of previously activated $\mathrm{T}$ cells (bystander activation).

These pathogenetic mechanisms are not mutually exclusive and may be particularly relevant at different stages of disease development. For instance, molecular mimicry could trigger the initial activation of autoreactive $T$ cells and/or induce expansion of a memory $T$ cell population, while superantigens could reactivate autoreactive $\mathrm{T}$ cells and induce relapses. Epitope spreading may be particularly relevant in diseases caused by chronic infection of the target organ, and in the chronic stage of an autoimmune process.

Immune responses that are directed against persistent infectious agents, and not against self-antigens, can also cause tissue damage. For example, infection with a noncytolytic virus renders cells susceptible to lysis by $\mathrm{CD}^{+} \mathrm{T}$ cells. Bacterial structures that persist for a relatively long time can elicit a chronic inflammatory response, as discussed below in the context of reactive arthritis. A strict definition of autoimmunity would exclude such diseases, because $T$ cells or antibodies specific for self-antigens are not responsible for tissue damage. However, in practice it can be difficult to make a clear distinction.

Table 1

Mechanisms for activation of autoreactive $T$ and $B$ cells by infectious agents

Molecular mimicry Viral and bacterial superantigens

Enhanced processing and presentation of autoantigens Bystander activation Activation of lymphocytes by lymphotropic viruses
Activation of autoreactive $T$ cells by microbial peptides that have sufficient structural similarity to self-peptides Activation of autoreactive $T$ cells that express particular $V \beta$ segments

Enhanced presentation of autoantigens by antigen-presenting cells recruited to an inflammatory site, followed by priming of autoreactive lymphocytes

Expansion of previously activated $T$ cells at an inflammatory site

Viral infection of lymphocytes, such as infection of B cells with hepatitis $C$ virus, resulting in enhanced antibody production and formation of circulating immune complexes 
Molecular mimicry. The initial expansion of naive autoreactive $T$ cells requires activation of the TCR by MHC-bound peptides or CD1-bound lipids/glycolipids. The molecular mimicry hypothesis proposes that microbial peptides with sufficient sequence similarity to selfpeptides can activate such T cells (2). Such sequence similarities were initially identified by homology searches (2), and more recently with search algorithms that consider the structural requirements for $\mathrm{T}$ cell receptor (TCR) recognition of MHC-bound peptides (3).

The concept of molecular mimicry was first tested in an experimental animal model with a hepatitis B virus polymerase peptide in which six amino acids were identical to the encephalitogenic region of rabbit myelin basic protein (MBP). T cell reactivity to MBP was observed following immunization of rabbits with this peptide, and four of eleven animals showed histological signs of experimental autoimmune encephalomyelitis (EAE) (2). This finding raised the important question of whether infection with viral or bacterial pathogens, rather than immunization with synthetic peptides, can also induce autoimmunity. This issue has now been addressed in a murine model of herpes simplex keratitis (HSK), a T cell-mediated inflammatory disease of the cornea that is induced by local application of herpes simplex virus (HSV). In humans, HSV-1-induced destruction of corneal tissue represents a leading cause of blindness. In the mouse model, keratogenic T cell clones induce disease following corneal application of the virus. These $\mathrm{T}$ cell clones crossreact with a peptide from the HSV-1 UL6 protein, and a virus with a mutated UL6 gene is greatly impaired in its ability to induce HSK (4). Together with more recent experiments employing a virus with a single amino acid mutation in the UL6 T cell epitope (5), these findings demonstrate that a viral infection can trigger $\mathrm{T}$ cell-mediated autoimmunity by molecular mimicry.

Another example of molecular mimicry comes from a murine myocarditis model in which disease is induced with peptides from Chlamydia. In BALB/c mice, immunization with a 30 -amino acid peptide from the cardiac myosin heavy chain induces a severe inflammatory heart disease. Peptides from the $60-\mathrm{kDa}$ cysteine-rich outer membrane protein of Chlamydia trachomatis and other Chlamydia species have sequence similarity with this myosin peptide and induce inflammatory heart disease at a similar frequency as the myosin peptide, although with a significantly lower severity. T cells from mice immunized with the Chlamydia peptide show a strong proliferative response to the myosin peptide, and such Chlamydia-reactive $\mathrm{T}$ cell lines induce moderately severe myocarditis. Bachmeier et al. (6) have observed that Chlamydia infection in mice results in the production of antibodies that crossreact with myosin, but the authors did not report whether such an infection induces myocarditis.

A new animal model of molecular mimicry was recently reported in which recombinant Theiler's viruses were generated by insertion of short segments that encoded for 30-amino acid peptides. This model was first tested with a virus that expressed a self-peptide from proteolipid protein (PLP, residues 139-151).
SJL mice infected with this neurotropic recombinant virus develop a rapid-onset paralytic disease characterized by a prominent $\mathrm{CD} 4^{+} \mathrm{T}$ cell response to the PLP peptide. Interestingly, a virus expressing a Hemophilus influenzae peptide that is recognized by PLP 139-151-specific T cells also causes disease; the disease induced by these recombinant viruses shows a much earlier onset than the chronic demyelinating disease observed with wild-type Theiler's virus. A major advantage of this approach is that a variety of potential mimicry peptides can be tested in vivo (7).

The majority of animal models that have examined the issue of TCR crossreactivity have focused on $\mathrm{CD}^{+}$ $\mathrm{T}$ cells. However, the role of $\mathrm{CD}^{+} \mathrm{T}$ cells has also been investigated in a mouse model of inflammatory bowel disease using $\mathrm{CD}^{+} \mathrm{T}$ cell clones that recognize both mycobacterial and murine hsp60. Adoptive transfer of hsp60-specific T cells into TCR $\beta^{-/}$mice leads to massive infiltration of these $T$ cells into the small intestine and the liver. Transfer of hsp60-specific T cells into wild-type mice does not cause such pathology, possibly because in vivo expansion of these $T$ cells is more limited in hosts that are not immunodeficient. Disease in this system is mediated by TCR recognition of the hsp60 self-antigen; a non-crossreactive $\mathrm{T}$ cell clone that only reacts with mycobacterial hsp60 does not cause disease. These results establish TCR crossreactivity between murine and bacterial hsp60 and indicate that molecular mimicry may also be relevant for $\mathrm{CD}^{+} \mathrm{T}$ cell populations (8).

Molecular mimicry is due to structural features of MHC/peptide/TCR complexes that limit TCR specificity for MHC-bound peptides (9). The structural basis of TCR crossreactivity has been defined using T cell clones from multiple sclerosis patients specific for an immunodominant peptide from human MBP. Such T cell clones recognize the MBP peptide bound to HLA-DR2, which is associated with susceptibility to multiple sclerosis (3). The crystal structure of the HLA-DR2 molecule with the bound MBP peptide shows that the two hydrophobic anchor residues of the MBP peptide are positioned in the hydrophobic $\mathrm{P} 1$ and P4 pockets of the binding site. The HLA-DR2 binding motif is highly degenerate since all peptide residues positioned in pockets of the HLA-DR2 binding site can be substituted by other amino acids $(3,10)$. Peptide elution studies have demonstrated that MHC molecules bind hundreds of different peptides (20).

In addition, only a limited number of peptide residues are important for TCR recognition. In the crystal structure, these peptide residues are located in the center of the HLA-DR2/MBP peptide surface (10). Accordingly, we searched databases of human pathogens for peptides that match the HLA-DR2 binding/TCR recognition motif and examined such peptides for their ability to activate human MBP-specific $\mathrm{T}$ cell clones. Using this approach we identified stimulatory microbial peptides for five of seven MBP-specific $T$ cell clones that were tested. A total of seven viral and six bacterial peptides were identified, indicating that MBP 85-99-specific $T$ cells could be activated by different pathogens $(3,11)$. 
Interestingly, only one of these peptides has obvious sequence similarity with the MBP peptide. The other microbial peptides are quite distinct in their sequence from the MBP peptide and from each other. Since the HLA-DR2 binding motif is highly degenerate, mimicry peptides require little or no homology with MBP 85-99 at the MHC interface. At the TCR contact surface, sequence homology or identity is limited to two or three peptide positions $(3,11)$.

Microbial peptides that activate another human MBPspecific $\mathrm{T}$ cell clone have been identified by first characterizing the $\mathrm{T}$ cell recognition motif with combinatorial peptide libraries. Using this strategy, the same MBP peptide residues were found to be important for $T$ cell recognition. Peptide recognition by this $\mathrm{T}$ cell clone is highly degenerate since random peptide libraries, which contain large numbers of different peptides $\left(\sim 2 \times 10^{14}\right.$ different sequences for an X11 library), stimulate this $\mathrm{T}$ cell clone (12). Several other relevant examples of TCR crossreactivity have been described and will be discussed in the section on human autoimmune diseases.

Viral and bacterial superantigens. Superantigens activate $\mathrm{T}$ cells through the variable domain of the TCR- $\beta$ chain. This distinctive mode of $\mathrm{T}$ cell activation, together with the ability of superantigens to bind to a wide variety of MHC class II molecules, leads to activation of large numbers of $\mathrm{T}$ cells irrespective of their MHC/peptide specificity. Superantigens are involved in several human diseases, including food poisoning and toxic shock syndrome (13).

Experiments in murine models of autoimmunity have clearly demonstrated that superantigens can induce relapses and exacerbations of a $\mathrm{T}$ cell-mediated autoimmune process. EAE can be induced in PL/J mice with the N-terminal peptide of MBP (Ac1-11). The majority of $\mathrm{T}$ cells specific for this peptide express V $\beta 8$, allowing activation of such $T$ cells by the superantigen staphylococcal enterotoxin B. Administration of this superantigen causes relapses and exacerbation of EAE (14), but it has not been possible to induce EAE by superantigen administration. On the contrary, injection of superantigen prior to immunization with MBP prevents the development of EAE in $\mathrm{PL} / \mathrm{J}$ mice due to deletion of $T$ cells that express $V \beta 8$ (15).

Superantigens can also trigger the reactivation of bacterial cell wall or collagen-induced arthritis. The Mycoplasma arthritidis superantigen (MAM) is derived from a naturally occurring murine arthritogenic mycoplasma and is a potent superantigen for V $\beta 5.1$-, $V \beta 6-$, and V $\beta 8$-positive T cells. MAM causes severe exacerbation of arthritis that persists for at least 40 days when administered during the chronic stage of the disease. The arthritis flare induced by MAM can be even more severe than the initial arthritis induced by type II collagen. The superantigen can also trigger arthritis in mice that did not develop clinical disease following the initial immunization with type II collagen (16). Viral and bacterial superantigens may therefore contribute to established autoimmune processes and induce relapses and exacerbations of disease.

Recently, a bacterial superantigen was isolated that may be important in the pathogenesis of Crohn dis- ease, a chronic inflammatory disease of the small intestine. Representational difference analysis resulted in the identification of microbial DNA sequences that were present in lesions, but absent from surrounding normal tissue. One of these genes encodes a bacterial transcription factor (termed I2) that is also present in murine intestine. The I2 protein induces vigorous proliferation of murine $\mathrm{V} 5^{+} \mathrm{CD} 4^{+} \mathrm{T}$ cells from nonimmunized mice. I2 protein fits the definition of a superantigen, since $T$ cell activation depends on MHC class II expression, but not on antigen processing (17).

Enhanced processing and presentation of autoantigens during an infection. A $T$ cell response directed against a single self-peptide can diversify during an inflammatory process by priming of $\mathrm{T}$ cells specific for other self-peptides. This concept of "epitope spreading" was first delineated in a murine EAE model. At an early time point following immunization with MBP (day 9), the $T$ cell response in both draining lymph nodes and spleen is focused on the N-terminal peptide of MBP (Ac1-11). However, at a later stage (day 40), $\mathrm{T}$ cell responses to several other MBP epitopes (residues 35-47, 81-100, and 121-140) can be detected. Importantly, epitope spreading is also observed in mice immunized only with the Ac1-11 peptide, indicating that endogenous priming to the self-antigen can lead to a diversification of the T cell response (18).

The role of epitope spreading in chronic viral infections has been examined in the Theiler's virus model (19). Theiler's murine encephalomyelitis virus, a natural mouse pathogen, is a picornavirus that induces a chronic, $\mathrm{CD}^{+} \mathrm{T}$ cell-mediated demyelinating disease. The virus persists in the CNS, and virus-specific $\mathrm{CD}^{+}$ $T$ cells initiate the demyelinating process. Clinical disease begins approximately 30 days after infection and displays a chronic-progressive course, with $100 \%$ of animals affected by $40-50$ days. T cell proliferative responses to virus can be detected in the spleen at the onset of clinical signs, but $T$ cell responses to myelin antigens are also observed at later stages of the disease. $T$ cell responses to an immunodominant peptide from PLP (residues 139-151) are detected first, followed by responses to other peptides derived from PLP, MBP, and myelin oligodendrocyte glycoprotein. Disease can be prevented by tolerance induction with viral peptides but not myelin peptides, indicating that virus-specific $T$ cells are key effector cells. Even though myelin-specific $T$ cells are not essential in the pathogenesis of this disease, the data clearly demonstrate that a chronic CNS infection can result in priming to self-antigens (19).

Bystander activation. Bystander activation has been thought to occur during viral infections due to local production of cytokines. Limiting dilution analyses, which were traditionally used to estimate the frequency of virus-specific $T$ cells, suggested that only a small fraction of activated $\mathrm{T}$ cells in viral infections are actually virus-specific. With the development of tetramer and intracellular cytokine staining techniques it has become apparent that the limiting dilution analyses greatly underestimated the actual frequency of virus-specific $T$ cells. Using these new techniques it has become apparent that the majority of activated $\mathrm{T}$ cells in viral infec- 
Table 2

Human inflammatory diseases induced by defined infectious agents

\begin{tabular}{|c|c|c|c|}
\hline Diseases & Major target organs & Pathogens & MHC Associations \\
\hline \multicolumn{4}{|l|}{ Postinfectious syndromes } \\
\hline Guillain-Barré syndrome & Peripheral nerve & $\begin{array}{l}\text { Campylobacter jejuni } \\
\text { Epstein-Barr virus } \\
\text { Cytomegalovirus }\end{array}$ & \\
\hline Rheumatic fever & $\begin{array}{l}\text { Heart muscle, heart valves } \\
\text { Kidney, CNS }\end{array}$ & Group A streptococci & \\
\hline \multicolumn{4}{|c|}{ Acute and chronic inflammatory diseases } \\
\hline Lyme arthritis & Large joints & Borrelia burgdorferi & HLA-DR4, HLA-DR1 \\
\hline Reactive arthritis & Axial skeleton & $\begin{array}{c}\text { Yersinia } \\
\text { Shigella } \\
\text { Salmonella } \\
\text { Chlamydia trachomatis }\end{array}$ & HLA-B27 \\
\hline \multicolumn{4}{|c|}{ Immune complex-mediated disease } \\
\hline Mixed cryoglobulinemia & $\begin{array}{l}\text { Blood vessels } \\
\text { Kidney, lung }\end{array}$ & Hepatitis C virus & \\
\hline
\end{tabular}

majority of autoimmune diseases, alleles of $\mathrm{MHC}$ class II genes show the strongest association. Since MHC class II molecules present peptides to $\mathrm{CD}^{+}{ }^{+} \mathrm{T}$ cells, these associations indicate that antigen presentation to $\mathrm{CD}^{+} \mathrm{T}$ cells may be important in the initiation and/or progression of these diseases. Notable exceptions are ankylosing spondylitis and reactive arthritis, which show a striking association with the MHC class I molecule HLA-B27 (24).

Criteria for establishing a role of infectious agents in autoimmune diseases. The concept of infectious triggers of human autoimmune dis-

tions are indeed antigen-specific (21). Since activation of naive $T$ cells requires signaling through the TCR, it is unlikely that bystander activation is responsible for the initial activation of autoreactive T cells. Enhanced local cytokine production could, however, lead to further expansion of previously activated T cells.

Activation of lymphocytes by lymphotropic viruses. Infection of human B cells by hepatitis $C$ virus can cause a lymphoproliferative disease, termed mixed cryoglobulinemia (MC). Infection of B cells results in B cell proliferation, enhanced antibody production, and the formation of circulating immune complexes (22). The clinical features and pathophysiology of this disease are described in more detail below. This example illustrates how a persistent virus infection of lymphocytes can result in an immune-mediated disease.

\section{Role of infectious agents in human inflammatory diseases}

The importance of genetic susceptibility. Genetic susceptibility to autoimmunity in humans and experimental animal models is due to the presence of multiple disease loci (23). Since particular combinations of genes confer susceptibility, only a relatively small fraction of the population appears to be genetically susceptible to a given autoimmune disease. Therefore, a particular autoimmune disease may only develop in a small number of individuals who encounter a certain infectious agent. The epidemiology of several human autoimmune diseases that are associated with defined infectious agents supports this concept, as discussed below (Table 2).

The MHC is an important susceptibility locus in many human autoimmune diseases, as well as in a number of experimental models $(23,24)$. The role of the $\mathrm{MHC}$ was first deduced from studies comparing the frequency of particular MHC alleles in patient and control populations. More recently, genetic linkage to the MHC was shown in genome-wide analyses of families with particular autoimmune diseases. In the eases has attracted considerable interest. However, clearcut criteria are required to establish a causative role for infectious agents in a disease process (Table 3 ). It is essential to isolate the infectious agent from patients with the disease and to demonstrate IgM antibodies to the infectious agent, which indicate recent exposure. In autoimmune diseases associated with acute infections it is critical to analyze appropriate control groups, such as household and community controls. As discussed below, these criteria have been successfully applied to the analysis of Guillain-Barré syndrome, an acute inflammatory disease of the peripheral nervous system.

For direct isolation of an infectious agent from patients with an autoimmune disease, a clinical diagnosis has to be made while the infectious agent is still present. This requirement can be met in acute autoimmune diseases that bring the disease to immediate medical attention and in chronic diseases that are caused by a persisting pathogen. However, isolation of an infectious agent can be difficult when the disease onset is slow and insidious, since the infectious agent may have been cleared prior to clinical diagnosis.

If an infectious agent is found to be clearly associated with an autoimmune process, it is important to define the mechanisms of disease pathogenesis. It is particularly relevant to determine whether the disease was initiated or amplified by the infectious agent and whether $T$ cells and/or antibodies mediate the disease. Analysis of such disease mechanisms can be greatly aided if animal models are available or can be developed. The following clinical examples will focus on diseases for which a definitive association with one or several infectious agents has been established.

Autoimmune diseases triggered by acute infections. Guillain-Barré syndrome (GBS) and rheumatic fever are classic examples of autoimmune diseases with an acute onset that follow infection with defined microorganisms. Rheumatic fever is triggered by infection with group A streptococci and affects multiple organs, in 
particular the heart, joints, kidney, and CNS. GBS is an inflammatory disease of the peripheral nervous system that can follow infection with Campylobacter jejuni, Epstein-Barr virus, cytomegalovirus, and Mycoplasma pneumoniae. Both examples highlight general principles of autoimmunity triggered by infection.

GBS is characterized by lymphocytic infiltration and demyelination in the peripheral nervous system. Typically, the onset is sudden and limb weakness progresses to maximum disability within 1 week of onset. In about $25 \%$ of cases, artificial ventilation is required because respiratory muscles are severely affected. Approximately two-thirds of patients report preceding illnesses, such as respiratory or gastrointestinal infections. The acute onset and the severity of the illness have greatly facilitated the isolation of infectious agents from patients with GBS (25). If the disease onset were slow and insidious as in some other autoimmune diseases, it would have been difficult to establish the association between GBS and these infectious triggers.

C. jejuni is the principal infectious agent that has been associated with the development of GBS. Campylobacter, the most common cause of bacterial diarrhea in the US, are Gram-negative bacilli that have a propensity to invade the intestinal mucosa. In GBS, the involvement of C. jejuni has been documented not only by serological methods, but also by direct isolation of the bacterium from GBS patients. In a well-controlled study that compared the frequency of positive cultures from patients and household controls, C. jejuni was found in $26 \%$ of GBS patients and $2 \%$ of household controls (26). Since such culture methods tend to underestimate the frequency of infection, a larger fraction of cases may be caused by this pathogen. Moreover, C. jeju$n i$ is an important worldwide cause of GBS, since studies conducted on four continents confirm its association with the disease (25). Summer epidemics of GBS occur among children and young adults in northern China and are particularly likely to be associated with C. jejuni infection (27). In Japan, a high prevalence of a particular serotype of C. jejuni (O:19) was observed among GBS patients (28). Different serotypes have been found in GBS patients in other countries.

Infection with $C$. jejuni induces antibodies that crossreact with peripheral nerve antigens. A number of studies have demonstrated that patients with GBS develop antibodies specific for LPS of certain strains of C. jejuni that crossreact with gangliosides from peripheral nerves. Gangliosides are membrane-anchored glycosphingolipids with a hydrophilic extracellular oligosaccharide. The outer polysaccharide moieties of LPS from certain strains of Campylobacter bear striking structural similarities to gangliosides found in peripheral nerves. For example, the Campylobacter O:19 serotype shares an identical tetrasaccharide with the $\mathrm{GM}_{1}$ ganglioside and a pentasaccharide with the $\mathrm{GD}_{1 \mathrm{a}}$ ganglioside. Serotypes O:23 and O:36 share a branched tetrasaccharide with the $\mathrm{GM}_{2}$ ganglioside (29).
Infection with C. jejuni also correlates with clinical features and the specificity of crossreactive autoantibodies. GBS following infection with C. jejuni is associated with a more severe clinical course, prominent motor symptoms, and the presence of antibodies to the $\mathrm{GM}_{1}$ ganglioside (26). In contrast, infection with cytomegalovirus is associated with a more pronounced sensory involvement, a milder clinical course, and antibodies that bind to the $\mathrm{GM}_{2}$ ganglioside (30). A strong association is observed with antibodies specific for ganglioside $\mathrm{GQ}_{1 \mathrm{~b}}$ and a clinical variant of GBS (Miller Fisher syndrome). GQ ${ }_{1 b}$ is concentrated in extraocular nerves, a principal motor site affected in this syndrome $(25,26,31)$.

Taken together, these data demonstrate a strong association between preceding infection with C. jejuni and the development of GBS. Nevertheless, susceptibility of the host is likely to play an important role in the development of this disease. The Center for Disease Control estimated that there are about 1000 cases of $C$. jejuni infection per 100,000 population per year, and only a small fraction of these cases develop GBS (incidence of approximately 1 per 100,000 population) (32). This situation is similar in other autoimmune diseases triggered by infections, such as rheumatic fever triggered by group A streptococci. It would therefore be of interest to define genes that confer susceptibility to GBS and to determine how these genes affect the immune response to Campylobacter.

In addition, very little is known about the $\mathrm{T}$ cell response to C. jejuni in GBS. In particular, it will be important to determine whether bacterial antigens activate $T$ cells that crossreact with peripheral nerve antigens. CD1 molecules can present bacterial glycolipids to $\mathrm{T}$ cells, raising the question whether peripheral nerve gangliosides could be recognized by CD1restricted $\mathrm{T}$ cells (33). It will also be important to develop an animal model in which inflammation and demyelination in the peripheral nervous system are induced by Campylobacter infection or by immunization with defined Campylobacter antigens. Defining the specificity of the $\mathrm{T}$ cell response in this disease may be critical in such efforts.

Triggering of rheumatic fever by group A streptococci. Rheumatic fever following pharyngeal infection with group A streptococci is another classic example of a postinfection autoimmune disease. The association of group A streptococci with rheumatic fever is strong, since outbreaks of rheumatic fever closely follow epidemics of streptococcal sore throats. Adequate treat-

\section{Table 3}

Criteria for establishing the role of infectious agents in autoimmune diseases

\section{Identification of pathogen(s) in patients with autoimmune disease}

Isolation of pathogen, which requires diagnosis of autoimmune process at the time of infection Analysis of appropriate control groups (household and community controls)

Analysis of $\mathrm{IgM}$ antibodies specific for pathogen

Determination of the mechanisms by which pathogen(s) induce autoimmunity

Analysis of T cell- and B cell-mediated immune responses to pathogen and potential self-antigens Development of an animal model that recapitulates essential features of the disease process 
ment of documented streptococcal pharyngitis markedly reduces the incidence of subsequent rheumatic fever. In addition, the recurrence of the disease can be prevented with antimicrobial prophylaxis. Due to widespread use of antibiotics, the disease has become rare in the US, but it is still common in developing countries $(34,35)$.

Typically, after an acute streptococcal pharyngitis there is a latent period of $2-3$ weeks, which is followed by an acute febrile illness that can involve the heart, joints, and/or CNS. Involvement of the heart valves is the most serious aspect of the disease and can result in severe functional impairment. Apparently due to differences between streptococcal strains that colonize mucus membranes and the skin, rheumatic fever only follows streptococcal pharyngitis but not streptococcal skin infections. Streptococci can also trigger a postinfectious glomerulonephritis in which immune complexes are deposited in the kidney. It has been suggested that different strains of Streptococcus are responsible for these different clinical outcomes $(34,35)$.

The streptococcal $\mathrm{M}$ protein is thought to play a role in the pathogenesis of rheumatic fever. $\mathrm{M}$ protein has an extended, $\alpha$-helical structure and has significant sequence homology with several human proteins, such as the myosin heavy chain, tropomyosin, laminin, and keratin. Human and murine antibodies that are specific for streptococcal $\mathrm{M}$ protein were found to crossreact with cardiac myosin. Antibody crossreactivity between $\mathrm{M}$ protein and cardiac myosin has been most extensively examined, but crossreactivity with the other structurally related proteins may also be relevant (36).

As in GBS, relatively little is known about the specificity of $T$ cells to streptococcal antigens in rheumatic fever, and there is no good animal model for the disease at the present time. Nevertheless, the epidemiological association of the pathogen with the disease is compelling, as are the isolation of the pathogen from patients with rheumatic fever, and the fact that this postinfectious syndrome can be prevented by early use of antibiotics $(34,35)$.

$\mathrm{CD}^{+} \mathrm{T}$ cells in the pathogenesis of Lyme arthritis. A chronic inflammatory joint disease is a complication of Lyme disease, which is caused by infection with the spirochete Borrelia burgdorferi. Susceptibility to Lyme arthritis is associated with alleles of MHC class II genes, notably HLA-DR4 and HLA-DR1, indicating that $\mathrm{CD}^{+} \mathrm{T}$ cells may be involved in the disease process. Lyme disease occurs worldwide, with most cases in temperate regions, and the earliest manifestation is an erythema migrans, which appears at the site of a deer tick bite. In some untreated individuals, the spirochete disseminates hematogenously to multiple sites. Symptoms of hematogenous dissemination include secondary skin lesions, mild hepatitis, cardiac disease, and neurological abnormalities. Arthritis and neurological disease dominate later phases of the illness (37). In the US, arthritis is the dominant feature of late Lyme disease, reported in about $70 \%$ of untreated individuals. In adults, symptoms range from intermittent to chronic arthritis, primarily in large joints. In the majority of patients, the arthritis can be successfully treated with antibiotics. However, about $10 \%$ of patients develop a treatment-resistant chronic arthritis that lasts for months or years (38-40).

The development of treatment-resistant arthritis could be due to persistent bacteria/bacterial antigens or due to the development of autoimmunity. Patients with persistent arthritis have negative findings for $B$. burgdorferi on PCR testing of joint fluid after more than 2 months of oral antibiotic therapy. Symptoms therefore persist after the apparent eradication of live spirochetes from joints with antibiotic therapy (38). However, it is possible that bacterial antigens persist for a longer period of time than live spirochetes.

Susceptibility to the treatment-resistant form of Lyme arthritis is associated with particular alleles of MHC class II genes, suggesting that $\mathrm{CD} 4^{+} \mathrm{T}$ cells play an important role in the pathogenesis. An increased frequency of HLA-DR4 (DRB1*0401) and HLA-DR1 (DRB1*0101 and DRB1*0102) is found in patients who have had arthritis for $1-4$ years (40). Interestingly, the same MHC class II alleles are associated with susceptibility to rheumatoid arthritis, a common inflammatory joint disease that is not associated with $B$. burgdorferi infection.

Severity and duration of Lyme arthritis correlate with the $\operatorname{IgG}$ antibody response to the outer surface proteins $\mathrm{A}$ and $\mathrm{B}$ (OspA and $\mathrm{OspB}$ ) of B. burgdorferi. These findings also support the hypothesis that $\mathrm{CD} 4^{+} \mathrm{T}$ cells are involved in the pathogenesis. A direct analysis of the $\mathrm{T}$ cell response to OspA demonstrated that treatment-resistant patients had an increased responsiveness to five different Ospa peptides, compared with patients who responded to treatment with antibiotics. Increased $\mathrm{T}$ cell responses were observed in blood and synovial fluid, indicating that both systemic and local immune responses to the bacteria differ between the two groups (39).

An immunodominant peptide of OspA (residues 164-183) is presented by HLA-DR4, which is associated with susceptibility to treatment-resistant Lyme disease. Search of protein sequence databases showed significant homology between this OspA peptide and human LFA-1. Gross et al. (41) reported that T cells from six of twelve patients with treatment-resistant Lyme disease showed a strong response to the OspA peptide, and $\mathrm{T}$ cells from four of these patients crossreacted with LFA-1. LFA-1 is not a joint-specific antigen, but the chronic inflammatory process results in the recruitment to the joints of large numbers of LFA-1-expressing lymphocytes (41). It will be important to determine whether $B$. burgdorferi-specific $T$ cells that crossreact with LFA-1 or other self-antigens are required for the development of treatment-resistant Lyme arthritis. Transgenic mice that express HLA-DR4 and human LFA-1 may help to address this question.

$C D 8^{+} T$ cells in the pathogenesis of reactive arthritis. Susceptibility to reactive arthritis, an acute inflammatory joint disease triggered by intestinal infection with certain bacteria, is associated with alleles of MHC class I genes, notably HLA-B27. The disease follows infection with intracellular bacteria, including Chlamydia, Salmonella, Shigella, and Yersinia species. The association of these bacteria with reactive arthritis is well established, 
based on isolation of bacteria and analysis of antibody responses. These bacteria enter the body through mucosal surfaces and are capable of invading living cells. For instance, Yersinia enterocolitica is taken up by $\mathrm{M}$ cells in Peyer's patches through an interaction between the bacterial surface protein invasin and host $\beta_{1}$-integrins. Yersinia can use phagocytes to translocate through endothelial monolayers, allowing it to enter the bloodstream and reach synovial tissue (42).

Reactive arthritis and ankylosing spondylitis have a strong association with the MHC class I allele HLA-B27. HLA-B27 is found in about $80 \%$ of cases with reactive arthritis, more than $95 \%$ of cases with primary ankylosing spondylitis, and about $9 \%$ of the general population. Ankylosing spondylitis is a chronic inflammatory joint disease that can be preceded by reactive arthritis. In Caucasian populations, ankylosing spondylitis is a relatively common disease, with a prevalence ranging from $0.1 \%$ to $0.8 \%$. Initial symptoms of ankylosing spondylitis are lower back pain and morning stiffness, which progress, often with exacerbations and remissions, over the years to a fixed rigidity of the spine. Ankylosing spondylitis can also show extraskeletal manifestations, such as acute anterior uveitis, aortic valve disease, and enteric mucosal inflammatory lesions. In most cases, ankylosing spondylitis is not associated with other disorders, but secondary ankylosing spondylitis sometimes occurs in association with reactive arthritis, psoriasis, ulcerative colitis, or Crohn disease $(42,43)$

Several lines of evidence indicate persistence of bacteria and/or bacterial antigens in patients with reactive arthritis. These patients have continued antibody responses against the triggering microorganisms, in particular IgA responses. Mononuclear phagocytes that carry antigens of arthritogenic microorganisms (LPS, heat shock proteins) can enter the peripheral circulation. It is thought that monocytes harboring such bacteria are the major source of microbial antigens that reach the synovium. In Yersinia-induced arthritis, LPS, $60-\mathrm{kDa}$ heat shock protein, and urease $\beta$-subunit have been detected in the joint by immunohistochemistry or immunoblotting. However, DNA from Yersinia has not been detected by PCR in joints. In contrast, chlamydial DNA and RNA are often detectable in the synovial membrane or synovial fluid (42).

The bacteria that cause reactive arthritis can replicate inside host cells, and their antigens can therefore be presented by the MHC class I pathway. HLA-B27-specific $\mathrm{T}$ cell responses have been demonstrated in the synovial fluid from patients with reactive arthritis, and HLA-B27-restricted peptide epitopes have been identified for the $60-\mathrm{kDa}$ heat shock protein and the urease $\beta$-subunit of Yersinia. Integrin $\alpha_{4} \beta_{7}$, which is known to be involved in the specific homing of $\mathrm{T}$ cells to the intestinal lamina propria and to the Peyer's patches, is also expressed by cells from synovial tissue $(42,44,45)$.

An interesting animal model of reactive arthritis has been generated by overexpression of HLA-B27. Such HLA-B27 transgenic mice and rats develop chronic inflammatory arthritis. Interestingly, the development of disease is dependent on the intestinal flora since no arthritis is observed under germ-free housing conditions. Therefore, disease development in this model may also be dependent on intestinal infection by certain bacteria $(46,47)$.

The hypothesis that reactive arthritis is due to a $\mathrm{T}$ cell response against persistent bacterial antigens in the joint does not readily explain the strong association of disease susceptibility with HLA-B27. A major question in the field is therefore whether an autoimmune response to synovial antigens plays a role in the disease process. Several alternative hypotheses have been proposed, which have been the subject of excellent reviews $(42,43)$.

Hepatitis $C$ virus and mixed cryoglobulinemia. $\mathrm{MC}$ is a systemic autoimmune disease caused by vascular deposition of circulating immune complexes and complement. Its name is based on the in vitro observation that immune complexes precipitate from the serum when it is cooled below $37^{\circ} \mathrm{C}$; these precipitates redissolve when the sample is brought back to $37^{\circ} \mathrm{C}$. The fact that a chronic hepatitis is observed in almost two-thirds of the patients led to the identification of hepatitis $C$ virus as a causative agent in the majority of patients with MC (22).

Hepatitis $C$ virus infects both hepatocytes and $B$ cells, due to binding of the $\mathrm{E} 2$ envelope protein to CD81 on the surface of hepatocytes and B cells (48). Infection of $B$ cells by hepatitis $C$ virus results in a lymphoproliferative disease with clonal expansion of $B$ cells. Hepatitis $C$ virus RNA is markedly more concentrated in the cryoprecipitate than in the supernatant, suggesting a direct involvement of hepatitis $C$ virus antigens in the immune complex-mediated vasculitis. Patients with $\mathrm{MC}$ also develop autoantibodies, due to $\mathrm{B}$ cell activation by the virus. Vascular deposition of these circulating immune complexes causes a vasculitis of small to medium-sized blood vessels and a nonerosive arthritis. Glomerulonephritis and alveolitis are consequences of deposition of immune complexes on basement membranes in the kidney and the lung. These data demonstrate that a virus can cause a systemic immune-mediated disease by activation of B cells (22).

\section{Future directions}

These clinical examples illustrate the relationship between infectious agents and the development of autoimmune diseases. In many of these diseases, we need to learn more about mechanisms of pathogenesis, based on the principles outlined at the beginning of this review. In particular, analysis of $\mathrm{T}$ cell responses with new techniques such as tetramers of MHC/peptide complexes and intracellular cytokine staining will be relevant. Humanized mouse models, such as MHC and TCR transgenic mice, may become valuable in the analysis of such $\mathrm{T}$ cell responses in vivo. For a number of common autoimmune diseases little is presently known about a potential role of infections. Prospective studies in subjects who carry a high risk for the development of such diseases may help to advance our knowledge of infectious triggers.

A better understanding of the relationship between infection and autoimmunity may allow prevention of autoimmune sequelae in some of these conditions. The 
case of rheumatic fever caused by group A streptococci demonstrates that it is possible to prevent the development of postinfectious autoimmunity by early intervention $(34,35)$.

1. Zamvil, S.S., and Steinman, L. 1990. The T lymphocyte in experimental allergic encephalomyelitis. Annu. Rev. Immunol. 8:579-621.

2. Fujinami, R.S., and Oldstone, M.B. 1985. Amino acid homology between the encephalitogenic site of myelin basic protein and virus: mechanism for autoimmunity. Science. 230:1043-1045.

3. Wucherpfennig, K.W., and Strominger, J.L. 1995. Molecular mimicry in $\mathrm{T}$ cell-mediated autoimmunity: viral peptides activate human $\mathrm{T}$ cell clones specific for myelin basic protein. Cell. 80:695-705.

4. Zhao, Z.S., Granucci, F., Yeh, L., Schaffer, P.A., and Cantor, H. 1998. Molecular mimicry by herpes simplex virus-type 1: autoimmune disease after viral infection. Science. 279:1344-1347.

5. Panoutsakopoulou, V., et al. 2001. Analysis of the relationship between viral infection and autoimmune disease. Immunity. 15:137-147.

6. Bachmaier, K., et al. 1999. Chlamydia infections and heart disease linked through antigenic mimicry. Science. 283:1335-1339.

7. Olson, J.K., Croxford, J.L., Calenoff, M.A., Dal Canto, M.C., and Miller, S.D. 2001. A virus-induced molecular mimicry model of multiple sclerosis. J. Clin. Invest. 108:311-318.

8. Steinhoff, U., et al. 1999. Autoimmune intestinal pathology induced by hsp60-specific CD8 T cells. Immunity. 11:349-358.

9. Wucherpfennig, K.W. 2001. Structural basis of molecular mimicry. J. Autoimmun. 16:293-302.

10. Smith, K.J., Pyrdol, J., Gauthier, L., Wiley, D.C., and Wucherpfennig, K.W. 1998. Crystal structure of HLA-DR2 (DRA*0101, DRB1*1501) complexed with a peptide from human myelin basic protein. J. Exp. Med. 188:1511-1520.

11. Hausmann, S., Martin, M., Gauthier, L., and Wucherpfennig, K.W. 1999. Structural features of autoreactive TCR that determine the degree of degeneracy in peptide recognition. J. Immunol. 162:338-344.

12. Hemmer, B., et al. 1997. Identification of high potency microbial and self ligands for a human autoreactive class II-restricted T cell clone. J. Exp. Med. 185:1651-1659.

13. Scherer, M.T., Ignatowicz, L., Winslow, G.M., Kappler, J.W., and Marrack, P. 1993. Superantigens: bacterial and viral proteins that manipulate the immune system. Annu. Rev. Cell Biol. 9:101-128.

14. Brocke, S., et al. 1993. Induction of relapsing paralysis in experimental autoimmune encephalomyelitis by bacterial superantigen. Nature. 365:642-644.

15. Soos, J.M., Schiffenbauer, J., and Johnson, H.M. 1993. Treatment of PL/J mice with the superantigen, staphylococcal enterotoxin B, prevents development of experimental allergic encephalomyelitis. J. Neuroim munol. 43:39-43.

16. Cole, B.C., and Griffiths, M.M. 1993. Triggering and exacerbation of autoimmune arthritis by the Mycoplasma arthritidis superantigen MAM. Arthritis Rheum. 36:994-1002.

17. Dalwadi, H., Wei, B., Kronenberg, M., Sutton, C.L., and Braun, J. 2001. The Crohn's disease-associated bacterial protein $\mathrm{I} 2$ is a novel enteric $\mathrm{T}$ cell superantigen. Immunity. 15:149-158.

18. Lehmann, P.V., Forsthuber, T., Miller, A., and Sercarz, E.E. 1992. Spreading of T-cell autoimmunity to cryptic determinants of an autoantigen. Nature. 358:155-157.

19. Miller, S.D., et al. 1997. Persistent infection with Theiler's virus leads to CNS autoimmunity via epitope spreading. Nat. Med. 3:1133-1136.

20. Hunt, D.F., et al. 1992. Characterization of peptides bound to the class I MHC molecule HLA-A2.1 by mass spectrometry. Science. 255:1261-1263.

21. Murali-Krishna, K., et al. 1998. Counting antigen-specific CD8 T cells: a reevaluation of bystander activation during viral infection. Immunity. 8:177-187.

22. Ferri, C., and Zignego, A.L. 2000. Relation between infection and autoimmunity in mixed cryoglobulinemia. Curr. Opin. Rheumatol. 12:53-60.
23. Vyse, T.J., and Todd, J.A. 1996. Genetic analysis of autoimmune disease. Cell. 85:311-318.

24. Nepom, G.T. 1993. MHC and autoimmune diseases. Immunol. Ser. 59:143-164.

25. Hughes, R.A., and Rees, J.H. 1997. Clinical and epidemiologic features of Guillain-Barré syndrome. J. Infect. Dis. 176(Suppl. 2):S92-S98.

26. Rees, J.H., Soudain, S.E., Gregson, N.A., and Hughes, R.A. 1995. Campylobacter jejuni infection and Guillain-Barré syndrome. N. Engl. J. Med. 333:1374-1379.

27. McKhann, G.M., et al. 1993. Acute motor axonal neuropathy: a frequent cause of acute flaccid paralysis in China. Ann. Neurol. 33:333-342.

28. Saida, T., et al. 1997. Campylobacter jejuni isolates from Japanese patients with Guillain-Barré syndrome. J. Infect. Dis. 176(Suppl. 2):S129-S134.

29. Moran, A.P. 1997. Structure and conserved characteristics of Campylobacter jejuni lipopolysaccharides. J. Infect. Dis. 176(Suppl. 2):S115-S121.

30. Visser, L.H., et al. 1996. Cytomegalovirus infection and Guillain-Barré syndrome: the clinical, electrophysiologic, and prognostic features. Dutch Guillain-Barré Study Group. Neurology. 47:668-673.

31. Carpo, M., et al. 1999. Clinical presentation and outcome of GuillainBarré and related syndromes in relation to anti-ganglioside antibodies. J. Neurol. Sci. 168:78-84.

32. Blaser, M.J. 1997. Epidemiologic and clinical features of Campylobacter jejuni infections. J. Infect. Dis. 176(Suppl. 2):S103-S105.

33. Porcelli, S.A., Segelke, B.W., Sugita, M., Wilson, I.A., and Brenner, M.B. 1998. The CD1 family of lipid antigen-presenting molecules. Immunol. Today. 19:362-368.

34. Gibofsky, A., and Zabriskie, J.B. 1995. Rheumatic fever and poststreptococcal reactive arthritis. Curr. Opin. Rheumatol. 7:299-305.

35. Stollerman, G.H. 1997. Rheumatic fever. Lancet. 349:935-942.

36. Cunningham, M.W., et al. 1989. Human and murine antibodies crossreactive with streptococcal $M$ protein and myosin recognize the sequence GLN-LYS-SER-LYS-GLN in M protein. J. Immunol. 143:2677-2683.

37. Evans, J. 1999. Lyme disease. Curr. Opin. Rheumatol. 11:281-288.

38. Carlson, D., et al. 1999. Lack of Borrelia burgdorferi DNA in synovial samples from patients with antibiotic treatment-resistant Lyme arthritis. Arthritis Rheum. 42:2705-2709.

39. Chen, J., et al. 1999. Association of antibiotic treatment-resistant Lyme arthritis with $T$ cell responses to dominant epitopes of outer surface protein A of Borrelia burgdorferi. Arthritis Rheum. 42:1813-1822.

40. Steere, A.C., Dwyer, E., and Winchester, R. 1990. Association of chronic Lyme arthritis with HLA-DR4 and HLA-DR2 alleles. N. Engl. J. Med. 323:219-223.

41. Gross, D.M., et al. 1998. Identification of LFA-1 as a candidate autoantigen in treatment-resistant Lyme arthritis. Science. 281:703-706.

42. Märker-Hermann, E., and Höhler, T. 1998. Pathogenesis of human leukocyte antigen B27-positive arthritis. Information from clinical materials. Rheum. Dis. Clin. North Am. 24:865-881.

43. López-Larrea, C., González, S., and Martínez-Borra, J. 1998. The role of HLA-B27 polymorphism and molecular mimicry in spondylarthropathy. Mol. Med. Today. 4:540-549.

44. Hermann, E., Yu, D.T., Meyer zum Büschenfelde, K.H., and Fleischer, B. 1993. HLA-B27-restricted CD8 T cells derived from synovial fluids of patients with reactive arthritis and ankylosing spondylitis. Lancet. 342:646-650

45. Ugrinovic, S., Mertz, A., Wu, P., Braun, J., and Sieper, J. 1997. A single nonamer from the Yersinia $60-\mathrm{kDa}$ heat shock protein is the target of HLA-B27-restricted CTL response in Yersinia-induced reactive arthritis. J. Immunol. 159:5715-5723.

46. Hammer, R.E., Maika, S.D., Richardson, J.A., Tang, J.P., and Taurog, J.D. 1990. Spontaneous inflammatory disease in transgenic rats expressing HLA-B27 and human $\beta_{2} \mathrm{~m}$ : an animal model of HLA-B27-associated human disorders. Cell. 63:1099-1112.

47. Taurog, J.D., et al. 1994. The germfree state prevents development of gut and joint inflammatory disease in HLA-B27 transgenic rats. J. Exp. Med. 180:2359-2364.

48. Pileri, P., et al. 1998. Binding of hepatitis C virus to CD81. Science. 282:938-941. 